\title{
Food-dependent drug-induced angioedema: a case report
}

\author{
Celso Eduardo Olivier ${ }^{* 1}$, Raquel Acácia Pereira Gonçalves dos Santos ${ }^{1}$, Daiana Guedes Pinto Argentão ${ }^{1}$, Mariana Dias da Silva ${ }^{1}$ and Regiane \\ Patussi dos Santos Lima ${ }^{1}$
}

${ }^{1}$ Instituto Alergoimuno de Americana Address: Rua Cuba, 836 - Americana, São Paulo, Brazil

\section{Introduction}

Food allergy (FA) is a complex condition that may reveal itself by unequivocal symptoms immediately after the ingestion of the food allergen, or it may persist asymptomatically until revealed by a metabolic alteration, such as the exercise [1,2]. In a similar way, adverse reactions to drugs may be just evident after the simultaneous contact with specific allergens such as the mite's constituents [3].

We report a case of FA whose symptoms were just revealed after the concomitant administration of the offending food and a nonsteroidal anti-inflammatory drug (NSAID). A 34-year-old Brazilian woman presented with a 10-years history of recurrent episodes of acute facial angioedema appearing predominantly some minutes after the use of NSAIDs (acetylsalicylic acid, ibuprofen and diclofenac). The first episode occurred after previous use of a flu vaccine. She referred that during the childhood and adolescence she had used NSAIDs without any adverse reaction and that occasionally she was able to tolerate some NSAIDs that were previously associated with the acute angioedema episodes. The episodes were severe, requiring the help of an emergency service to receive rescue medication (steroids and antihistamines), with resolution $36-48$ hours after medication. The emergence doctors summarily diagnosed the illness as "drug allergy" and oriented the patient to change the NSAIDs. She did not present any respiratory symptoms and was not suspicious of any food allergy, but complained of recurrent headaches that had been investigated by a neurologist without a definitive diagnosis. We proceed to perform a panel of allergic skin tests to evaluate food allergy as well the drugs associated with the angioedema episodes [4]. The skin allergy tests demonstrated immediate reactions (wheal/flare) to histamine (14/21 $\mathrm{mm})$ and to hen's egg white $(13 / 17 \mathrm{~mm})$. The skin allergy tests to the allergen's diluent (negative control), NSAIDs drugs (acetylsalicylic acid, ibuprofen, diclofenac, acetaminophen and meloxicam), inhalants and others aliments were not reactive. The patient related that she never associated any symptoms with the use of hen's egg and ate them regularly at her meals. We performed an oral drug challenge test with meloxicam that reproduced her symptoms (facial angioedema) after the dose of $5 \mathrm{mg}$ was achieved. The patient was medicated with fexofenadine and prednisone, oriented to avoid food prepared with hen's egg, and asked to collect lab's exams. She returned after three weeks with a total IgE level of $97 \mathrm{kIU} / \mathrm{L}$ and a hen's egg white-specific immunoglobulin E level of $3.4 \mathrm{IU} / \mathrm{L}$ (ImmunoCAP). She related to be adhering to the exclusion diet and was not in use of any medication. We proceed to another oral drug challenge test with ibuprofen, achieving progressively the dose of $600 \mathrm{mg}$ without adverse effects. After a week, we scheduled to perform an oral food challenge with hen's egg white. She was able to tolerate cooked hen's egg white of three eggs without any reaction. This challenge was performed in the morning. In the afternoon of the same day, she presented headache and took a dose of ibuprofen. After some minutes, she developed facial angioedema and returned to us to receive rescuing medication. Therefore, we realized that the NSAIDs adverse reactions just occurred if the patient had previously ingested hen's egg white. In fact, the patient became able to use any NSAIDs (including meloxicam) if she had not ingested previously anything with hen's egg white. She related further that with the exclusion diet, her headaches disappeared.

Most NSAIDs exert their effect by the inhibition of the activity of the cyclooxygenase enzymes: cyclooxigenase-1 (COX-1) and cyclooxigenase-2 (COX-2), and thereby, the synthesis of eicosanoids such as the prostaglandins and thromboxanes [5]. The eicosanoids are not stored by the cells, but rather produced after a proper stimulus. The substrate for this synthesis is the arachidonic acid released from the cellular membrane by the cytosolic phospholipases activated by several mechanisms such as physical stimuli, bacterial constituents, the interaction of some hormones and autacoids with membrane receptors and the increase of cytosolic calcium as the consequence of the mast cell degranulation promoted by IgE crosslink [6]. Once available in cytosol, the arachidonic acid is quickly oxidized by several distinct enzymatic systems such as the cyclooxygenases and the lipoxygenases. In short, the cyclooxygenases pathway generate pro-inflammatory autacoids such as the prostaglandins and thromboxanes; while the lipoxygenases pathway generates the leukotrienes, a group of autacoids with a strong effect on postcapillary venules and smooth musculature, resulting in increased extravascular exudation and muscle constriction respectively [5,7]. When the activity of the phospholipases is increased for whatsoever reason, the inhibition of the cyclooxygenases promoted by NSAIDs provides to the lipoxygenase pathway an additional amount of arachidonic acid resulting in an increased production of leukotrienes, what explain the collateral effects such as the angioedema $[8,9]$.

We conclude that adverse effects to NSAIDs may be dependent of a concomitant food allergy and therefore, food allergy must be investigated in patients with adverse reactions to NSAIDs.

\section{Clinical implications}

Adverse reactions to non-steroidal anti-inflammatory drugs may be an indicative of food allergy.

Correspondence to: Olivier CE, Instituto Alergoimuno de Americana Address: Rua Cuba, 836 - Americana, São Paulo, Brazil

Key words: food hypersensitivity, drug hypersensitivity, anti-inflammatory agents, non-steroidal

Received: March 05, 2017; Accepted: March 20, 2017; Published: March 24 2017 


\section{Declaration of all sources of funding}

Olivier CE, Argentão DGP, Lima RPS, Silva MD and Santos RAPG received grant support from Instituto Alergoimuno de Americana.

\section{Competing interests}

The authors declare that they have no competing interests.

\section{References}

1. Morita E, Kunie K, Matsuo H (2007) Food-dependent exercise-induced anaphylaxis. $J$ Dermatol Sci 47: 109-117. [Crossref]

2. Olivier CE (2013) Food Allergy. Journal of Allergy \& Therapy 1-7.

3. Sánchez-Borges M, Fernández-Caldas E, Capriles-Hulett A, Caballero-Fonseca F (2012) Mite-induced inflammation: More than allergy. Allergy Rhinol (Providence) 3: e25-29. [Crossref]
4. Olivier CE, Argentão DGP, Santos RAPG, Silva MD, Lima RPS, et al. (2013) Skin scrape test: an inexpensive and painless skin test for recognition of immediate hypersensitivity in children and adults. The Open Allergy Journal 6: 9-17.

5. Smith WL, Garavito RM, DeWitt DL (1996) Prostaglandin endoperoxide H synthases (cyclooxygenases)-1 and -2. J Biol Chem 271: 33157-33160. [Crossref]

6. Shimizu T (2009) Lipid mediators in health and disease: enzymes and receptors as therapeutic targets for the regulation of immunity and inflammation. Annu Rev Pharmacol Toxicol 49: 123-150. [Crossref]

7. Liu M, Yokomizo T (2015) The role of leukotrienes in allergic diseases. Allergol Int 64: 17-26. [Crossref]

8. Kowalski ML, Makowska JS, Blanca M, Bavbek S, Bochenek G, et al. (2011) Hypersensitivity to nonsteroidal anti-inflammatory drugs (NSAIDs) - classification, diagnosis and management: review of the EAACI/ENDA(\#) and GA2LEN/HANNA* Allergy 66: 818-829. [Crossref]

9. Chaudhry T, Hissaria P, Wiese M, Heddle R, Kette F, et al. (2012) Oral drug challenges in non-steroidal anti-inflammatory drug-induced urticaria, angioedema and anaphylaxis. Intern Med J 42: 665-671. [Crossref]

Copyright: $\odot 2017$ Olivier CE. This is an open-access article distributed under the terms of the Creative Commons Attribution License, which permits unrestricted use, distribution, and reproduction in any medium, provided the original author and source are credited. 